\title{
Myocardial Infarction Mortality in Rural and Urban Hospitals: Rethinking Measures of Quality of Care
}

\author{
Paul A. James, $M D^{1}$ \\ Pengxiang $L i, M A^{2}$ \\ Marcia M. Ward, $\mathrm{PbD}^{2}$ \\ 'Department of Family Medicine, Roy J. \\ and Lucille A. Carver College of Medicine, \\ University of Iowa, Iowa City, Iowa \\ ${ }^{2}$ Department of Health Management and \\ Policy, College of Public Health, University \\ of Iowa, lowa City, Iowa
}

\begin{abstract}
PURPOSE Patients with acute myocardial infarction have higher mortality rates in rural hospitals than in urban hospitals, suggesting substandard quality of care in the rural setting. We examined characteristics of patients experiencing myocardial infarction and used an instrumental variable technique to adjust for unmeasured confounding when comparing mortality rates for these hospitals.
\end{abstract}

METHODS We used the 2002 and 2003 lowa State Inpatient Datasets, including 12,191 lowa residents aged 18 years or older hospitalized with a principal diagnosis of acute myocardial infarction (International Classification of Diseases, Ninth Revision, Clinical Modification [ICD-9-CM] codes 410.01- 410.91) in 116 lowa hospitals classified as rural or urban. In-hospital mortality was the primary outcome measure. Age, sex, race, admission type, payer, and 2 comorbidity indices (Charlson Comorbidity Index and All Patient Refined Diagnosis-Related Groups) were determined to calculate risk-adjusted mortality. The distance from each patient's home to the nearest urban lowa hospital was used as an instrumental variable to compare risk-adjusted mortality controlled for unmeasured confounders.

RESULTS Unadjusted and risk-adjusted mortality rates using logistic regression models indicated significantly lower in-hospital mortality for patients with myocardial infarction admitted to urban hospitals than for their counterparts admitted to rural hospitals (unadjusted values, $6.4 \%$ vs $14 \%$ ). The urban and rural groups differed significantly on characteristics studied, however. Analyses indicated that the traditional logistic regression models were possibly confounded by unmeasured patient factors, and when the same data were analyzed with the instrumental variable technique, mortality differences disappeared.

CONCLUSIONS In lowa, mortality from myocardial infarction in rural hospitals is not higher than that in urban ones after controlling for unmeasured confounders. Current risk-adjustment models may not be sufficient when assessing hospitals that perform different functions within the health care system. Unmeasured confounding is a major concern when comparing heterogeneous and undifferentiated populations.

Ann Fam Med 2007;5:105-111. DOI: 10.1370/afm.625.

\section{INTRODUCTION}

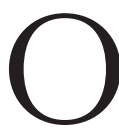
bservational studies suggest that the quality of care in rural hospitals is substandard for patients with myocardial infarction. ${ }^{1,2}$ Separately, other studies examining the effect of specialty care on mortality also attribute higher mortality to rural settings where specialist care is limited. ${ }^{3.7}$ These data support conventional wisdom because rural hospitals have lower volumes of patients with myocardial infarction than urban hospitals ${ }^{8}$; usually do not have cardiologists and therefore rely on generalists to care for these patients ${ }^{9}$ ire geographically inaccessible, causing patients to delay access after the onset of chest pain ${ }_{i}$ and lack the support services necessary for interventions shown to reduce mortality. ${ }^{10}$ 
The validity of these observational studies is increasingly questioned, however. ${ }^{11}$ Unmeasured confounding may somewhat account for the observed differences in patient outcomes. ${ }^{12,13}$ For example, usual practice in rural hospitals dictates transferring patients needing higher levels of care to regional referral hospitals or to urban hospitals where advanced technologies and services are available. ${ }^{14}$ Yet data show that patients admitted to rural hospitals tend to be older, poorer, and sicker, and have more comorbidities-all factors that contribute to increased mortality. 2,12,13 This pattern of selective admissions to rural hospitals appears counterintuitive as it suggests that rural practitioners send the youngest, healthiest patients with less severe myocardial infarction to urban hospitals and admit the oldest, sickest patients with myocardial infarction to rural hospitals. ${ }^{15}$ We suspect that previously unmeasured confounders may explain this unexpected pattern. We therefore compared mortality outcomes between patients with myocardial infarction admitted to rural vs urban hospitals, using observed in-hospital mortality, risk-adjusted mortality, and mortality outcomes controlled for unmeasured confounders by the technique of instrumental variables.

\section{METHODS}

\section{Study Data}

Our analyses used 2 years of discharge data (2002 and 2003) from the State Inpatient Dataset of Iowa. Using International Classification of Diseases, Nintb Revision, Clinical Modification (ICD-9-CM) codes, we identified 15,081 patients aged 18 years or older who were hospitalized with a principal diagnosis of myocardial infarction (codes 410.01-410.91). We excluded cases wherein the hospital identification number was missing ( $\mathrm{n}=$ $9)$, the patient's ZIP code $(n=14)$ or sex $(n=1)$ was missing, and the patient's home county was not in Iowa $(\mathrm{n}=1,248)$. Patients transferred to another acute care general hospital for inpatient care $(n=1,618)$ were analyzed separately. Most of our analyses are based on 12,191 patients with myocardial infarction.

\section{Dependent and Independent Variables}

Iowa has 116 nonfederal hospitals that are classified as Urban Hospitals, Rural Referral Hospitals, Rural Prospective Payment System (PPS) Hospitals, or Critical Access Hospitals (CAHs). The 20 Urban Hospitals are located in a Metropolitan Statistical Area (MSA). The 7 Rural Referral Hospitals are located in nonMSA areas but have operating characteristics similar to those of urban hospitals. ${ }^{16}$ The $80 \mathrm{CAHs}$ became part of the Medicare Rural Hospital Flexibility Program, which permits them to bill for services to
Medicare beneficiaries in rural areas on a cost basis. The remaining 9 rural hospitals that have not converted to CAH status are now referred to as Rural PPS Hospitals because they continue to bill Medicare using Diagnosis-Related Groups (DRGs). A primary comparison in our analyses is between rural and urban hospitals. We combined CAHs and Rural PPS Hospitals to create the rural category, and we combined Rural Referral Hospitals and Urban Hospitals to create the urban category.

We specified binary variables for payer (Medicare, Medicaid, private insurance, self-pay, no charge, other), admission type (emergency, urgent, other), and race (black or not). We used 2 comorbidity indices: the Charlson Comorbidity Index ${ }^{17}$ and the All Patient Refined DRG (APR-DRG) risk index. The Charlson Comorbidity Index was created based on all secondary diagnoses. ${ }^{17,18}$ The APR-DRG classifies patients into 382 clinically meaningful groups. Within each group, patients were divided into 4 severity-of-illness and 4 risk-of-mortality subclasses. ${ }^{19}$ The APR-DRG risk index was created by using the entire 2002-2003 Iowa State Inpatient Dataset as a standard to estimate the probability of death for patients with each combination of APR-DRG values. ${ }^{20}$

The distances between each patient's home and all urban hospitals in lowa were obtained by calculating the distances between the centroids of each patient's residential ZIP code and all urban hospitals' ZIP codes The shortest distance was the patients' distance to the nearest urban hospital. Our outcome measure was inhospital mortality.

\section{Traditional Analytic Approach}

For the univariate analyses of group comparisons, $\chi^{2}$ tests were used for dichotomous data and analyses of variance were used for continuous data. To compare adjusted mortality outcomes between patients admitted to rural hospitals vs urban hospitals, we used logistic regression analyses and controlled for patient characteristics. We first adjusted for demographic characteristics (age, sex, race, admission type, and payer). Next, we included each comorbidity index (Charlson Comorbidity Index and APR-DRG risk index). We then estimated the in-hospital mortality of 2 subgroups of patients (admitted to rural hospitals or to urban hospitals) using the best logistic regression model and compared the goodness of fit of the models for the 2 populations using $c$ statistics and the HosmerLemeshow $\chi^{2}$ test. $^{21}$

\section{Instrumental Variable Approach}

The traditional risk-adjustment approach can only adjust for measured patient characteristics. If important 
characteristics associated with in-hospital mortality are not available in the data set and the omitted relevant characteristics are related to patient or physician selection of the hospital, the traditional approaches (eg, logistic regression analysis) may yield biased estimates of the impact of hospital type (urban vs rural) on in-hospital mortality. For example, the difference in mortality rate could be due to unmeasured patient risk factors instead of quality of care. In such cases, an instrumental variable technique has been recommended for its ability to adjust for potential unmeasured confounding effects. . $^{13,22-25}$

The instrumental variable method is an econometric technique that enables an unbiased estimation of treatment effects in observational studies. A detailed description of this technique is given in an article by Newhouse and McClellan. ${ }^{22}$ For elderly patients with myocardial infarction, several unmeasured confounders, such as severe comorbid conditions that limit life expectancy or patient preferences to remain in the rural hospital, may reduce transfers to larger urban hospitals and thereby increase mortality. As discussed above, the biases of traditional approaches are due to the correlation between the variable of interest (rurality in this case) and unmeasured confounders. The instrumental variable technique can extract variation in the variable of interest that is unrelated to unmeasured confounders, and use this variation to estimate the causal effect on an outcome. ${ }^{22-25}$

The instrumental variables should satisfy 2 assumptions: (1) they should correlate with treatment choice and (2) their effect on outcome should only be through treatment choice (ie, they should have no relationship with unmeasured confounders). ${ }^{22-25}$ Instrumental variables are used to achieve a "pseudorandomization." 21 The outcome of a coin toss in a randomized controlled trial would be a perfect instrumental variable, ${ }^{21}$ if we could use a coin toss to assign patients to rural or urban hospitals. In this case, a patient's choice of hospital would depend only on the outcome of the coin toss and would not be associated with any unmeasured confounders, such as the severity of myocardial infarction. We could therefore attribute the observed effects to the treatment and derive an unbiased estimate. In observational studies, distance is often used to create the instrumental variables for studying outcomes of myocardial infarction. ${ }^{13,22}$ Similar to the approach of Brooks et $\mathrm{al}^{23}$ instrumental variables in our study were binary variables that grouped patients based on their distance to the nearest urban hospital.

We hypothesized that the distance of a patient's home to the nearest urban hospital would independently predict the likelihood of selection of either a rural or an urban hospital but would not be related to the patients' comorbidities or other unmeasured confounders. For estimation, the SYSLIN 2-stage leastsquares (2SLS) procedure in SAS version 9.1 (SAS Institute, Cary, NC) was used. We used F statistics in the first-stage regression to test the first assumption of the instrumental variables. ${ }^{24,25}$ We examined the second assumption of the instrumental variables in several ways. As indicated by Newhouse and McClellan, ${ }^{22}$ if the instrumental variables are independent of the unmeasured confounders, it should also be independent of observed risk factors (eg, age and comorbidity index). Using approaches similar to those of Newhouse and McClellan, ${ }^{22}$ Frances et $\mathrm{al}_{1}{ }_{13}$ and Brooks et $\mathrm{al}_{,}{ }^{23}$ we tested the independence of the instrumental variables with observed risk factors. Following Brooks et al, ${ }^{23}$ instrumental variables were constructed as categorical variables by separating patients into $2,4,8$, and 12 groups based on the distance of a patient's home to the nearest urban hospital. In addition, overidentifying restrictions tests* were used to examine the second assumption of instrumental variables. ${ }^{24,25}$

\section{RESULTS \\ Patient Characteristics}

The crude in-hospital mortality rate was significantly higher for patients with myocardial infarction admitted to rural hospitals (14.0\%) than for their counterparts admitted to urban hospitals (6.4\%). As shown in Table 1 , the demographics and levels of comorbidity

* The null hypothesis for overidentifying restrictions tests is that instruments are uncorrelated with unmeasured confounders. ${ }^{25}$ 
of the 2 groups differed significantly. Specifically, patients admitted to urban hospitals were younger and more likely to be male and black. Although the groups did not differ in the number of secondary diagnoses, patients' Charlson Comorbidity Index and APR-DRG risk index were lower (indicating less severity) in urban hospitals than in rural ones. Patients with myocardial infarction admitted to rural hospitals were thus sicker and substantially older than their counterparts admitted to urban hospitals.

As a tangential analysis, we compared patients with myocardial infarction who were originally admitted to rural hospitals but then transferred to another acute care general hospital with patients with myocardial infarction who were admitted to rural hospitals and remained there. As shown in Table 2, those transferred to another acute care hospital were younger, were more likely to be male, and had fewer secondary diagnoses, a lower Charlson Comorbidity Index, and a lower APR-DRG risk index than those who stayed in the rural hospital. Patients who were originally admitted to a rural hospital but then transferred to another acute care hospital thus had a lower risk profile than those who stayed in rural hospitals.

Table 2. Baseline Characteristics of Patients With Myocardial Infarction Staying in Rural Hospitals or Transferring Out of Rural Hospitals

\begin{tabular}{lccc}
\hline Characteristic & $\begin{array}{c}\text { Stayed in Rural } \\
\text { Hospitals } \\
(\mathbf{n}=\mathbf{1 , 4 2 6 )}\end{array}$ & $\begin{array}{c}\text { Transferred } \\
\text { Out of Rural } \\
\text { Hospitals } \\
\mathbf{( n = 7 3 0 )}\end{array}$ & P Value \\
\hline Age, mean, years & 82.4 & 71.5 & $<.001$ \\
Male, \% & 45.0 & 56.7 & $<.001$ \\
Black, \% & 0.14 & 0.14 & .98 \\
Number of secondary diagnoses, mean & 5.66 & 4.24 & $<.001$ \\
Charlson Comorbidity Index, mean & 0.96 & 0.67 & $<.001$ \\
APR-DRG risk index, mean & 0.09 & 0.04 & $<.001$ \\
\hline APR-DRG = All Patient Refined Diagnosis-Related Group. & & \\
\hline
\end{tabular}

Table 3. Odds Ratios for In-Hospital Mortality Among Patients With Myocardial Infarction Admitted to Urban vs Rural Hospitals, Using Logistic Regression Models

\begin{tabular}{lccc}
\hline Logistic Regression Model & $\begin{array}{c}\text { OR (95\% CI) } \\
\text { (Urban vs Rural } \\
\text { Hospitals) }\end{array}$ & $\begin{array}{c}\text { P Value of } \\
\text { the Hospital } \\
\text { Variable }\end{array}$ & $\begin{array}{c}\text { C Statistic } \\
\text { Unadjusted }\end{array}$ \\
$\begin{array}{l}\text { Adjusted for demographic characteristics } \\
\text { (age, sex, race, admission type, payer) }\end{array}$ & $0.42(0.35-0.50)$ & $<.001$ & 0.56 \\
$\begin{array}{l}\text { Adjusted for demographic characteristics } \\
\text { and Charlson Comorbidity Index }\end{array}$ & $0.73(0.58-0.84)$ & $<.001$ & 0.70 \\
$\begin{array}{l}\text { Adjusted for demographic characteristics } \\
\text { and APR-DRG risk index }\end{array}$ & $0.68(0.56-0.82)$ & $<.001$ & 0.71 \\
\hline $\begin{array}{l}\text { Note: Excluding patients discharged or transferred to another acute care general hospital for inpatient care. } \\
\text { OR = odds ratio; Cl = confidence interval; APR-DRG = All Patient Refined Diagnosis-Related Group. }\end{array}$ \\
\hline
\end{tabular}

\section{Logistic Regression Analyses}

We used logistic regression analyses to compare mortality rates between patients with myocardial infarction who were admitted to and remained in rural vs urban hospitals. As shown in Table 3, all models showed that patients admitted to urban hospitals had a lower in-hospital mortality rate than their counterparts admitted to rural hospitals when using traditional risk-adjustment approaches. The best model in terms of the $c$ statistic was obtained using the demographic characteristics plus the APR-DRG risk index as covariates (Table 3 ).

We then estimated the in-hospital mortality of the 2 groups of patients with myocardial infarction (admitted to rural vs urban hospitals) using the best logistic regression model and examined the goodness of fit of the logistic regression models. The $c$ statistic was 0.88 for the urban hospital model and 0.69 for the rural hospital model (data not shown). The Hosmer-Lemeshow $\chi^{2}$ with 8 degrees of freedom was 11.56 for the urban hospital model and was not significant at the $5 \%$ level. It was 22.22 for the rural hospital model, which was significant at the $1 \%$ level. The logistic regression model for patients admitted to rural hospitals had a much worse goodness-of-fit statistic than the model for patients admitted to urban hospitals. This finding indicates that a substantial amount of variation in patients with myocardial infarction in rural hospitals cannot be captured in these multivariate models.

\section{Instrumental Variable Analyses}

The unbalanced patient characteristics between rural and urban hospitals, and the poor goodness of fit for the rural hospital model suggested that the traditional analytic approaches for comparing mortality caused by myocardial infarction may be subject to bias because of omitted variables. We tried to address this possible bias using instrumental variable methods. To evaluate the validity of the instrumental variable, we separated the patients with myocardial infarction into 2 groups based on their distance to the nearest urban hospital, using the median distance (14.08 miles) as the cut point. As shown in Table 4, patients who lived closer to an urban hospital were much 
more likely to be admitted to an urban hospital than those who lived farther away. Although the groups differed significantly in terms of age, the difference was much smaller than that between patients admitted to urban vs rural hospitals (Table 1 ). There were no significant differences between groups in the Charlson Comorbidity Index or the APR-DRG risk index. This comparison validated our assumption that the distance to the nearest urban hospital significantly influences the selection of either an urban or a rural hospital, and is not associated with patients' severity of diseases.

As shown in Table 5, the F test for the instrumental variable indicated that the distance to the nearest urban hospital substantially affected selection of hospital type for patients with myocardial infarction. If the F statistic for instrumental variables is less than 10 , the
Table 4. Baseline Characteristics Among Patients With Myocardial Infarction Grouped by Distance to the Nearest Urban Hospital

\begin{tabular}{|c|c|c|c|}
\hline \multirow[b]{2}{*}{ Characteristic } & \multicolumn{2}{|c|}{$\begin{array}{l}\text { Distance to Nearest } \\
\text { Urban Hospital }\end{array}$} & \multirow[b]{2}{*}{$P$ Value } \\
\hline & $\begin{array}{l}\leq 14.08 \text { Miles } \\
(n=6,097)\end{array}$ & $\begin{array}{l}>14.08 \text { Miles } \\
(n=6,104)\end{array}$ & \\
\hline $\begin{array}{l}\text { Distance to the nearest urban hospital, } \\
\text { mean, miles }\end{array}$ & 4.94 & 34.20 & $<.001$ \\
\hline Admitted to urban hospitals, \% & 99.54 & 77.09 & $<.001$ \\
\hline Age, mean, y & 68.89 & 72.02 & $<.001$ \\
\hline Male, \% & 58.65 & 57.45 & .18 \\
\hline Black, \% & 1.95 & 0.08 & $<.001$ \\
\hline Number of secondary diagnoses, mean & 5.72 & 5.53 & $<.001$ \\
\hline Charlson Comorbidity Index, mean & 0.72 & 0.72 & .67 \\
\hline APR-DRG risk index, mean & 0.07 & 0.07 & .48 \\
\hline Length of stay, d & 5.34 & 5.30 & .70 \\
\hline In-hospital mortality rate, \% & 7.07 & 7.52 & .34 \\
\hline
\end{tabular}

instrumental variables are weak, given that instrumental variables have small partial correlation with the variable of interest, and the 2SLS estimation is likely to be biased. ${ }^{24,26}$ Our instrument $\mathrm{F}$ statistics ranged from roughly 184 to 1,694 , indicating a moderate to strong influence of the instrumental variables. In the overidentifying restrictions tests, ${ }^{*}$ only 1 model rejected the null hypothesis of no correlation with unmeasured confounders, indicating the validity of the instrumental variables. The instrumental variable estimates consistently

* These tests can only be done if there is more than 1 instrumental variable. ${ }^{24,25}$

Table 5. Instrumental Variable Estimates of the Difference of In-Patient Mortality Between Urban and Rural Hospitals

\begin{tabular}{|c|c|c|c|c|c|}
\hline \multirow[b]{2}{*}{ Instrumental Variable Model } & \multirow{2}{*}{$\begin{array}{l}\text { Number of } \\
\text { Groups for } \\
\text { Instrumental } \\
\text { Variable }\end{array}$} & \multicolumn{2}{|c|}{$\begin{array}{l}\text { Tests for } \\
\text { Instrumental Variables }\end{array}$} & \multicolumn{2}{|c|}{$\begin{array}{l}\text { IV Estimates of Hospital } \\
\text { Type (Urban vs Rural) }\end{array}$} \\
\hline & & $\begin{array}{l}\text { Instrument } \\
\text { F Statistic* }\end{array}$ & $\begin{array}{c}P \text { Value for } \\
\text { Overidentifying } \\
\text { Restrictions Tests }{ }^{\dagger}\end{array}$ & Coefficient $\beta$ & $P$ Value \\
\hline \multirow[t]{4}{*}{ Unadjusted } & 2 & $1,540.16$ & - & -0.02 & .34 \\
\hline & 4 & 642.65 & .65 & -0.03 & .16 \\
\hline & 8 & 294.02 & .20 & -0.03 & .14 \\
\hline & 12 & 184.31 & .13 & -0.03 & .13 \\
\hline \multirow{4}{*}{$\begin{array}{l}\text { Adjusted for demographic characteristics } \\
\text { (age, sex, race, admission type, payer) }\end{array}$} & 2 & $1,568.24$ & - & 0.013 & .58 \\
\hline & 4 & 652.86 & .80 & 0.0081 & .69 \\
\hline & 8 & 289.74 & .22 & 0.0084 & .68 \\
\hline & 12 & 187.14 & .10 & 0.0065 & .75 \\
\hline \multirow{4}{*}{$\begin{array}{l}\text { Adjusted for demographic characteristics } \\
\text { and Charlson Comorbidity Index }\end{array}$} & 2 & $1,539.90$ & - & 0.0090 & .69 \\
\hline & 4 & 642.51 & .92 & 0.0053 & .80 \\
\hline & 8 & 289.72 & .25 & 0.0060 & .77 \\
\hline & 12 & 184.29 & .12 & 0.0040 & .84 \\
\hline \multirow{4}{*}{$\begin{array}{l}\text { Adjusted for demographic characteristics } \\
\text { and APR-DRG risk index }\end{array}$} & 2 & $1,694.27$ & - & -0.0034 & .87 \\
\hline & 4 & 640.61 & .92 & -0.0069 & .72 \\
\hline & 8 & 318.20 & .17 & -0.0032 & .87 \\
\hline & 12 & 202.50 & .01 & -0.0063 & .74 \\
\hline
\end{tabular}


showed that being admitted to urban hospitals was no longer associated with significantly lower in-hospital mortality in patients with myocardial infarction after controlling for the bias resulting from omitted variables. We also undertook Hausman tests, ${ }^{27}$ which suggested that the traditional estimations of hospital type (urban vs rural) were biased." We replicated the analyses among nontransferred patients with myocardial infarction (excluding those patients transferred from other acute care hospitals) and among the 3-year State Inpatient Dataset of Iowa (including years of 2001, 2002, and 2003) ${ }^{\dagger}$ The results were consistent with our results in Table $5 .^{\ddagger}$ Finally, we tested our data using the bivariate probit model for instrumental variable estimation, and the results we obtained were consistent with 2SLS estimations.

\section{DISCUSSION}

This study confirms earlier studies that showed patients with myocardial infarction admitted to rural hospitals were significantly older and sicker than their counterparts admitted to urban hospitals. ${ }^{2,12,13}$ Unadjusted and adjusted mortality rates obtained using traditional logistic regression models indicated significantly lower odds of in-hospital mortality for patients admitted to urban hospitals. Our findings suggest, however, that these models could be biased because admissions to rural or urban hospitals are likely to be confounded by unmeasured patient-level variables. This potential confounding was reflected in the poorer goodness-of-fit model for patients admitted to rural hospitals and the results of Hausman tests. ${ }^{27}$

The benefit of the instrumental variable approach is that one obtains unbiased and reasonably good estimates of the effect of the treatment on the outcome variable, if the instrumental variables chosen meet the 2 assumptions. ${ }^{21}$ We attempted to remove residual selection bias by choosing the distance to the nearest urban hospital from a patient's residence as an instrumental variable. ${ }^{13,22,23,28}$ We rigorously tested the instrumental variables and found them to be valid, and demonstrated that the instrumental variable estimates should be unbiased. We believe that this approach offers advantages because it yields unbiased estimation. It does so by comparing the mortality of patients with myocardial infarction between rural and urban hospitals among those comparable patients ${ }^{22,23}$ who selected the hospital for myocardial infarction care based on

* Given the word limit, the results are not reported. They are available on request from the authors.

† We did not run the APR-DRG risk adjusted models for 3-year data given that APRDRG variables were not available in 2001 data set.

$\neq$ These tests can only be done if there is more than 1 instrumental variable..$^{24,25}$ the fact that it was the geographically closest hospital. In other words, patients chose rural or urban hospitals because they lived near the hospitals. They would not have chosen these hospitals had they lived farther away. We believe that most patients with myocardial infarction select the hospital that is geographically nearby. Our findings from the instrumental variable estimation differ from the findings obtained with the logistic regression models and show that patients with myocardial infarction admitted to urban hospitals no longer have reduced in-hospital mortality compared with their counterparts admitted to rural hospitals.

Our results have potential limitations. First, the instrumental variable estimation can be generalized only to patients with myocardial infarction whose selection of admitting hospital was influenced by their geographic location. ${ }^{21,22}$ For example, the conclusion cannot be applied to patients with myocardial infarction in rural areas who bypass rural hospitals and seek care in urban hospitals. A second possible limitation is that the findings for hospitals in 1 state do not generalize to other states. Likewise, analyses of in-hospital mortality rates may not be generalizable to mortality rates after discharge. We recommend replication of this work with larger, national data sets.

Our results conflict with findings from previous observational studies. Health care professionals experienced in rural health care delivery know that the previous studies are inconsistent with established practice. Rural physicians in small hospitals with minimal supporting infrastructure do not admit, but rather transfer quickly patients with myocardial infarction who require intensive medical management. Yet the traditional analytic approaches suggest that the patients sent to urban hospitals tend to be younger and less severely ill than those who remain in the rural hospitals. This pattern of patient selection for urban hospitals suggests that the clinical judgment about transfer of rural elderly patients with myocardial infarction may rely on different criteria, and this influence likely contributes to the unmeasured confounding.

We believe that those different criteria are not reflected in the data sets we used for study and may not be uniformly reflected in patients' charts. The preferences of patients likely play a substantial role in hospital selection, especially among elderly patients experiencing myocardial infarction. Patient preferences may reflect personal choices or the existence of serious comorbid conditions. Patients who have complex medical and personal histories may choose to remain near home and ultimately to die near home. The transfer patterns may therefore reflect rural physicians respecting patients' decisions that are complex and not related to disease. 
Rural hospitals, because of their size and limited personnel, often function as triage hospitals. Our study provides evidence to support the continued importance of rural hospitals and their role in caring for patients with myocardial infarction.

This study adds to the questions previously raised about the use of in-hospital mortality rates as a quality indicator for hospitals, ${ }_{1}^{29}$ even when risk adjustment is used. Current methods to monitor quality may be flawed if they do not adequately control for selection bias caused by unmeasured confounders. By showing an important influence of such confounders, we believe that this study strongly challenges the contention that care for myocardial infarction is inferior in rural hospitals.

To read or post commentaries in response to this article, see it online at http://www.annfammed.org/cgi/content/full/5/2/105.

Key words: Myocardial infarction; mortality; rural hospitals; instrumental variable; cardiovascular diseases; health care delivery; quality of care; health services research; quantitative methods; confounders

Submitted March 23, 2006; submitted, revised, June 13, 2006; accepted July 24, 2006.

Funding support: Support for this work was provided by the Agency for Healthcare Research and Quality through grant HS015009.

Acknowledgment: The authors thank Mirou Jaana for assisting with the analyses of mileage.

\section{References}

1. Sheikh K, Bullock C. Urban-rural differences in the quality of care for Medicare patients with acute myocardial infarction. Arch Intern Med. 2001;161(5):737-743.

2. Baldwin LM, MacLehose RF, Hart LG, et al. Quality of care for acute myocardial infarction in rural and urban US hospitals. J Rural Health. 2004;20(2):99-108.

3. Frances CD, Go AS, Dauterman KW, et al. Outcome following acute myocardial infarction: are differences among physician specialties the result of quality of care or case mix? Arch Intern Med. 1999;159(13):1429-1436.

4. Ayanian JZ, Guadagnoli E, McNeil BJ, Cleary PD. Treatment and outcomes of acute myocardial infarction among patients of cardiologists and generalist physicians. Arch Intern Med. 1997;157(22):2570-2576.

5. Jollis JG, DeLong ER, Peterson ED, et al. Outcome of acute myocardial infarction according to the specialty of the admitting physician. N Engl J Med. 1996;335(25):1880-1887.

6. Nash IS, Corrato RR, Dlutowski MJ, O'Connor JP, Nash DB. Generalist versus specialist care for acute myocardial infarction. Am J Cardiol. 1999;83(5):650-654

7. Norcini JJ, Kimball HR, Lipner RS. Certification and specialization: do they matter in the outcome of acute myocardial infarction? Acad Med. 2000;75(12):1193-1198.

8. Jollis JG, Romano PS. Volume-outcome relationship in acute myocardial infarction: the balloon and the needle. JAMA 2000:284(24):3169-3171
9. Goldman L. The value of cardiology. N Engl J Med. 1996;335(25): 1918-1919.

10. Rosenblatt RA. Quality of care in the rural context: a proposed research agenda. J Rural Health. 2002;18(Suppl):176-185.

11. Hartz A, James PA. A systematic review of studies comparing myocardial infarction mortality for generalists and specialists: lessons for research and health policy. J Am Board Fam Med. 2006;19(3):291-302.

12. Chen J, Radford MJ, Wang Y, Krumholz HM. Care and outcomes of elderly patients with acute myocardial infarction by physician specialty: the effects of comorbidity and functional limitations. Am J Med. 2000;108(6):460-469.

13. Frances CD, Shlipak MG, Noguchi H, Heidenreich PA, McClellan M. Does physician specialty affect the survival of elderly patients with myocardial infarction? Health Serv Res. 2000;35(5 Pt 2):1093-1116.

14. Wakefield DS, Ward M, Miller T, et al. Intensive care unit utilization and interhospital transfers as potential indicators of rural hospital quality. J Rural Health. 2004;20(4):394-400.

15. Mehta RH, Stalhandske EJ, McCargar PA, Ruane TJ, Eagle KA. Elderly patients at highest risk with acute myocardial infarction are more frequently transferred from community hospitals to tertiary centers: reality or myth? Am Heart J. 1999;138(4 Pt 1):688-695.

16. lowa Hospital Association. Profiles. Des Moines, lowa: lowa Hospital Association; 2002

17. Charlson ME, Pompei P, Ales KL, MacKenzie CR. A new method of classifying prognostic comorbidity in longitudinal studies: development and validation. J Chronic Dis. 1987;40(5):373-383.

18. Deyo RA, Cherkin DC, Ciol MA. Adapting a clinical comorbidity index for use with ICD-9-CM administrative databases. J Clin Epidemiol. 1992;45(6):613-619.

19. Richard F, Muldoon J, Vertrees J, et al. The Evolution of Casemix Measurement Using Diagnosis Related Groups (DRGs). 3M HIS Research Report; 1998. Available at: http://www.yardleyconsulting. com/Download/evolcasemix5-98.pdf. Accessed: 8 April 2005.

20. Romano PS, Chan BK. Risk-adjusting acute myocardial infarction mortality: are APR-DRGs the right tool? Health Serv Res. 2000;34(7):1469-1489

21. Hosmer DJ, Lemeshow S. Applied Logistic Regression. New York, NY: John Wiley \& Sons; 1989.

22. Newhouse JP, McClellan M. Econometrics in outcomes research: the use of instrumental variables. Annu Rev Public Health. 1998;19:17-34.

23. Brooks JM, Chrischilles EA, Scott SD, Chen-Hardee SS. Was breast conserving surgery underutilized for early stage breast cancer? Instrumental variables evidence for stage II patients from lowa. Health Serv Res. 2003;38(6 Pt 1):1385-1402.

24. Stock J. Watson M. Introduction to Econometrics. 1st ed. Boston, Mass: Addison-Wesley; 2003.

25. Woolridge J. Econometric Analysis of Cross Section and Panel Data. Cambridge, Mass: The MIT Press; 2002.

26. Staiger D, Stock J. Instrumental variables regression with weak instruments. Econometrica. 1997;65(3):557-586.

27. Hausman J. Specification tests in econometrics. Econometrica. $1978 ; 46: 1251-1271$

28. Ayanian JZ, Landrum MB, Guadagnoli E, Gaccione P. Specialty of ambulatory care physicians and mortality among elderly patients after myocardial infarction. N Engl J Med. 2002;347(21):1678-1686.

29. Wennberg JE, Freeman JL, Shelton RM, Bubolz TA. Hospital use and mortality among Medicare beneficiaries in Boston and New Haven. N Engl J Med. 1989;321(17):1168-1173. 